\title{
Research and Application of Certificate Management System with Floating Window
}

\author{
Fengwei Wang ${ }^{1, a}$,Huan Zhang ${ }^{1, a}$, Na Liu $^{1, a}$, Yue Wang ${ }^{1, a}$ \\ ${ }^{1}$ Department of Quality and Technology, Liaoning Provincial Institute of Measurement, Shenyang, \\ 110004, China \\ aEmail: 250178551@qq.com
}

Keywords: Floating window; Certificate Approval; windows API; COM component

\begin{abstract}
Different with certificate approval on paper, online certificate approval is difficult to record approve information, operate complicated, find out the error location in the certificate and so on, so the applications don't work out well. This paper introduces the certificate approval way with floating window based on Windows API and Com component technology. With this system, when the approved by approve certificate, the floating window will hide automatically, and it will display in a small window when the approved find out error information. In order to find out error location easily, the verified by can double-click the error information in the float window which is easy to operate.
\end{abstract}

\section{Introduction}

The certificate document is the final product provided to the customer by the measurement technology. With the rapid increasing testing business, the measurement institutions have been trying to explore how to increase the efficiency of issuing, reviewing, printing certificates all the time. In order to realize the efficient and standardized management of certificates, it is necessary to realize the inwindowation management of certificates, and establish standardized information processing mechanism.

At present, a lot of testing and calibration laboratories in China have realized the information management of certificates. The management systems implemented with Word online editing, template application, electronic signature and other means, free the detection personnel from a number of tedious, repetitive work, which effectively improve the work efficiency. But the management information systems, without exception, have encountered the same problem in the application: it is complex to browse and label meanwhile, difficult to record the error information and correspond to the specific location of the document in question.

In Liaoning provincial institute of measurement, the number of calibration certificates is up to 200000 copies per year, with a per capita share of 2500 copies. In order to ensure the standard of the approval of certificates and to improve the work efficiency, we must use a list of advanced computer technologies to improve the ease of recording error information during approval certificate, such as COM components, secondary development of MS Word and other advanced computer methods, on the one hand, record approval information conveniently, on the other hand, the verified-by modify certificate according to the error information conveniently.

\section{Business Process Analysis}

Verified by need to test, make the calibration certificate, check the calibration certificate and approve every day. According to the role, the certificate of business management process is divided into verified by, checked by and approver. After carding, certificate management business flow chart is shown in figure 1. 


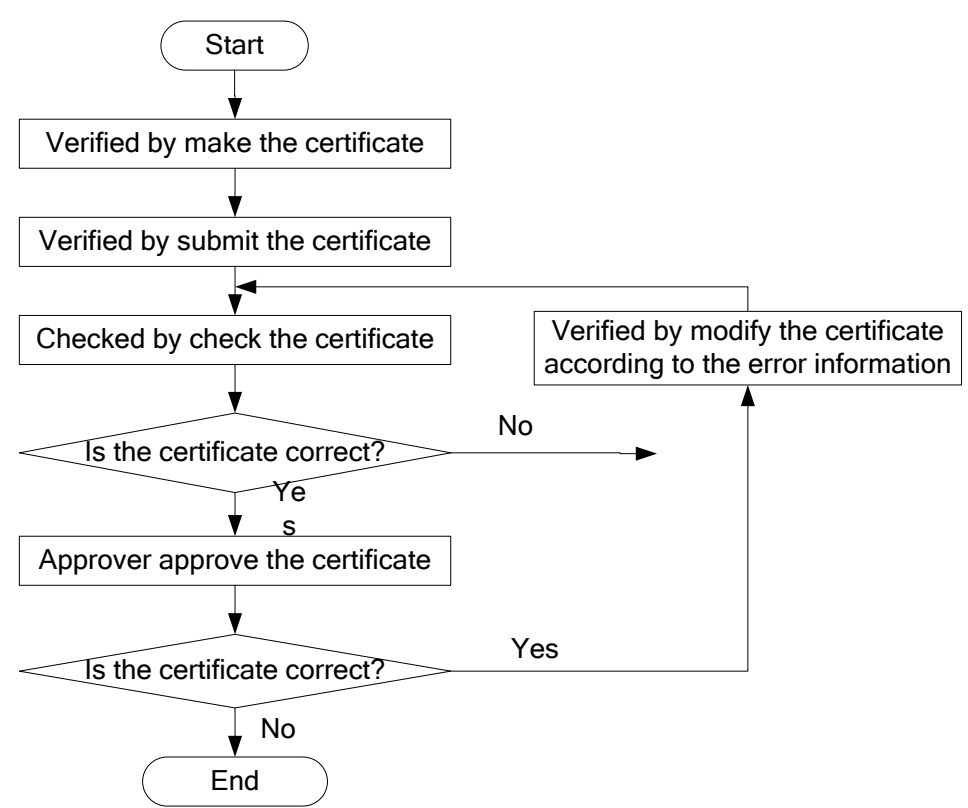

Fig.1. The business flow chart of certificate management

The certificate approval business process is described as follows:

(1) After the instrument has been checked, the verified by select the corresponding template according to the testing item and make the certificate, fill the test data to the certificate.

(2) The verified by submits the certificate to the corresponding checked by and approver.

(3) The checked by checks the certificate information and document content. If some mistakes are found, the checked by needs to record the opinion and rejects the certificate to the verified by; if there is no problem, the certificate is submitted to the approver automatically.

The approver approves the certificate information and document content. If some mistakes are found, the approver records the opinion and rejects the certificate to the verified by; if there is no problem, the electronic signatures are added automatically.

\section{System key technology}

\section{A Floating window based on windows API}

The floating window function is namely that the check/approve window can display on or hide behind the verification certificate automatically by moving the mouse on the center or edge of check/approve window. Taking the approving certificate window as an example, when the approver finds some mistakes, he moves the mouse on the window, the Windows operating system will automatically send a message to the application who can get the message with method "GetMessage () ", then the approve window will show according to the message accepted by method "WndProc ()"[1], so that the checked by or approver can record the mistake information directly and system records the error location automatically. After recording, approver moves the mouse to leave the window, the approve window will hide automatically so that he can continue to browse the certificate document.

This system is developed based on .NET Framework, Visual Studio 2005 development kits, and implements three states of the floating window by setting the "Top" and "Height" attributes of window:

(1) hidden state: does not show the check/approve certificate window (the window's "Top" property is less than 0 );

(2) small state: show the check/approve certificate window partly (the window's "Top" attribute is equal to 0 , the window's "Height" attribute value is equal to 100);

(3) complete state: display form completely (the window's "Top" attribute is equal to 0 , the window's "Height" attribute value is equal to the height of the window).

The small state of checking certificate window is shown in figure 2. 


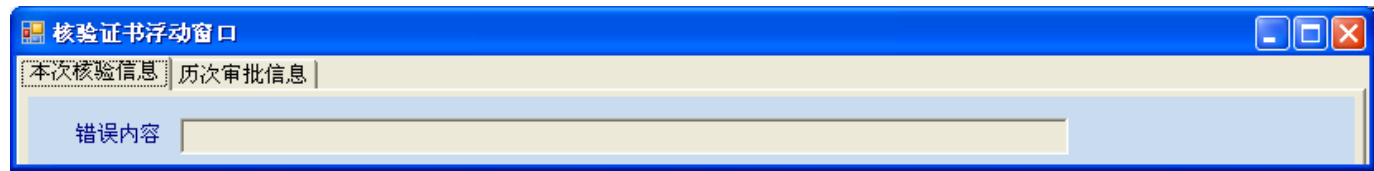

Fig.2 The small state of checking certificate window

The complete state of checking certificate window is shown in figure 3.

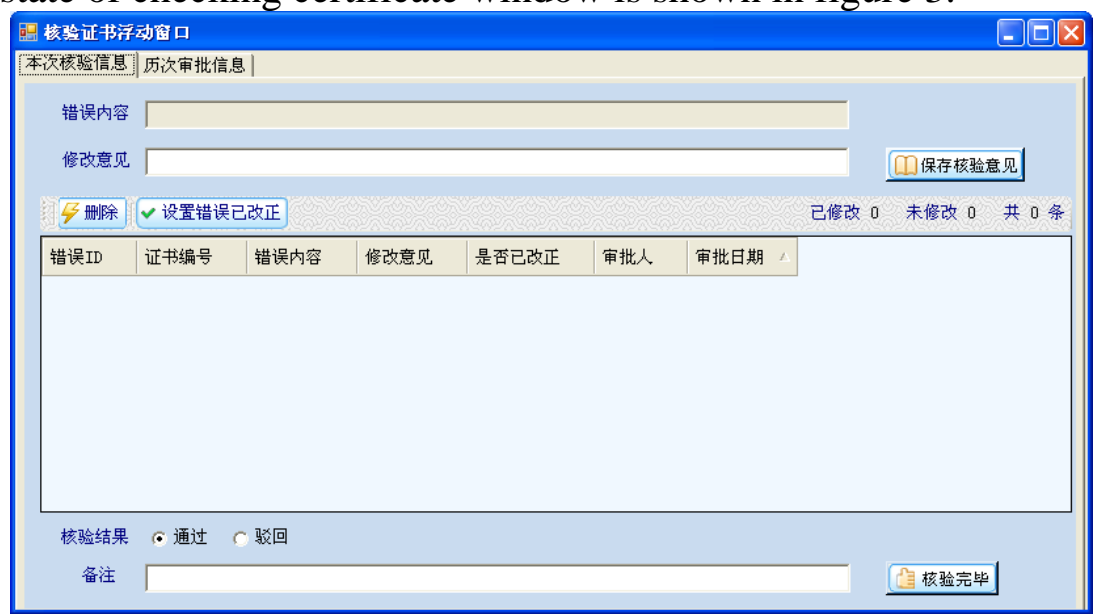

Fig.3 The complete state of checking certificate window

The application rewrites (override) method "WndProc", and WM_MOUSEMOVE (mouse moves on the window), WM_MOUSELEAVE (mouse leaves window) and WM_NCMOUSEMOVE (mouse moves over the title bar), WM_NCMOUSELEAVE (mouse leaves the title bar) and other events, so that the floating window can be set to different state[2].

With the floating window, checked by or approver can open the certificate document and window at the same time, floating window is set to small state default, which do not affect browsing certificate; with the mouse over a window into the system of floating form, floating window is set to complete state in order to record the opinion; After inputting the opinion, the mouse left the floating window, and then the floating window will be hidden automatically that the checked by or approver can browse certificate continually.

Word positioning technology based on COM component

At present, the certificate documents are stored as Word format in most of LIMS, therefore, each system must implement accessing to Word and second developing. This system implements with COM component "Primary Interop Assemblies" which makes the software components interact in a unified way[3].

In this system, the certificate template are stored on the file server in Word format. When the verified by need to make a certificate, he chooses a template from template library where each template had many bookmarks. The chose template is downloaded automatically and the detect information are filled on the template with each bookmark automatically, such as customer name, equipment name, norm type and others.

Bookmarks can be achieved in the Word document in different locations jump, fast positioning. The main steps for certificate generation are as follows:

(1) create Word.ApplicationClass object "WordApp”.

(2) when need to open the word document, call the method "WordApp.Documents.Open ()".

(3) when the document opened, use the "Range" property of the bookmark to locate it, and use the method "BookMarks.Add ()" to set the bookmark content to the text you want to display. After setup is complete, the document is open.

(4) when the verified by clicks "close" button on the Word document, the system call "Close ()" method.

(5) after the document is closed, read the property "WordApp.Documents.Count", if it is 0, indicating that the object control document has been completely closed, the object can be directly released.

If some mistakes are found during the checking or approving, the checked by or approver can 
select the error information in the document, then the system will automatically select the text (Range.Text) display in a floating window, and automatically record the selected content start position and end position (Range.Start) (Range.End)[4], and save to the database. In this way, when the verified by modifies the certificate has mistakes, the method "Range.Select ()" can be used to locate the error location in the document automatically[5], which is convenient to browse and modify.

The certificate error information saved in the database is shown in table 1.

Table 1 Error Information Saved in the Database

\begin{tabular}{|r|l|r|r|l|}
\hline ErrorID & ErrorInfo & StartLocation & EndLocation & Opinion \\
\hline 1040 & $250 \mathrm{~V}$ & 8127 & 8132 & $220 \mathrm{~V}$ \\
\hline 1041 & re1 & 8465 & 8468 & Should not use Italic \\
\hline 1042 & $0 \sim 20 \mathrm{~A}$ & 2720 & 2726 & $(0-20)$ A \\
\hline
\end{tabular}

\section{Conclusion}

This paper introduces a system with floating window, which does not affect the certificate document browsing, and convenient recording error information, at the same time, the error information to locate function also provides convenience for verified by modifying certificate. The system has been used for more than two years in the Liaoning Provincial Institute of Measurement, and has been recognized. The implementation and technical means can be provided to other measurement technology institutions for reference.

\section{References}

[1] Hu Zhi-wen, Chen Guo-long. Effective Consuming Windows API Functions with Visual Studio.NET[J], COMPUTER ENGINEERING AND APPLICATIONS, 2004, 40(12):108-111.

[2] Han Jianbin. Research on Windows API in VBA programming[J], Computer Era, 2012, (6): 13-14.

[3] ZHANG Jian-bing YANG Chong-jun LIU Dong-lin LE Xiao-qiu. Design and Implementation of Floating Window Map System [J], MINI-MICRO SYSTEMS, 2006, 27 (2): 287-290.

[4] Zhou Wen-jun, Liu Zhu-hong. Research on Word paper generation technology based on COM component [J], Computer Knowledge and Technology, 2005,11(24): 62-63,66.

[5] FU Yong-jie, CAI Ying, ZHANG Wei. Automatic generation of verification Certificate with VB Word-Oriented Programming[J], Industrial Measurement, 2009, 19(6):21-23. 